\title{
Relação entre a espessura corneana e a gravidade de defeito de campo visual no glaucoma primário de ângulo aberto
}

\author{
Relationship between corneal thickness and severity of visual field loss in primary \\ open-angleglaucoma
}

\author{
Sergio Henrique Sampaio Meirelles ${ }^{1}$ \\ Riani Morelo Álvares ${ }^{2}$ \\ Priscilla Barbuto Botelho ${ }^{3}$ \\ Fábio Barreto Morais ${ }^{4}$ \\ Pedro Bertino Moreira ${ }^{5}$ \\ Ana Carolina de Magalhães Villela ${ }^{6}$
}

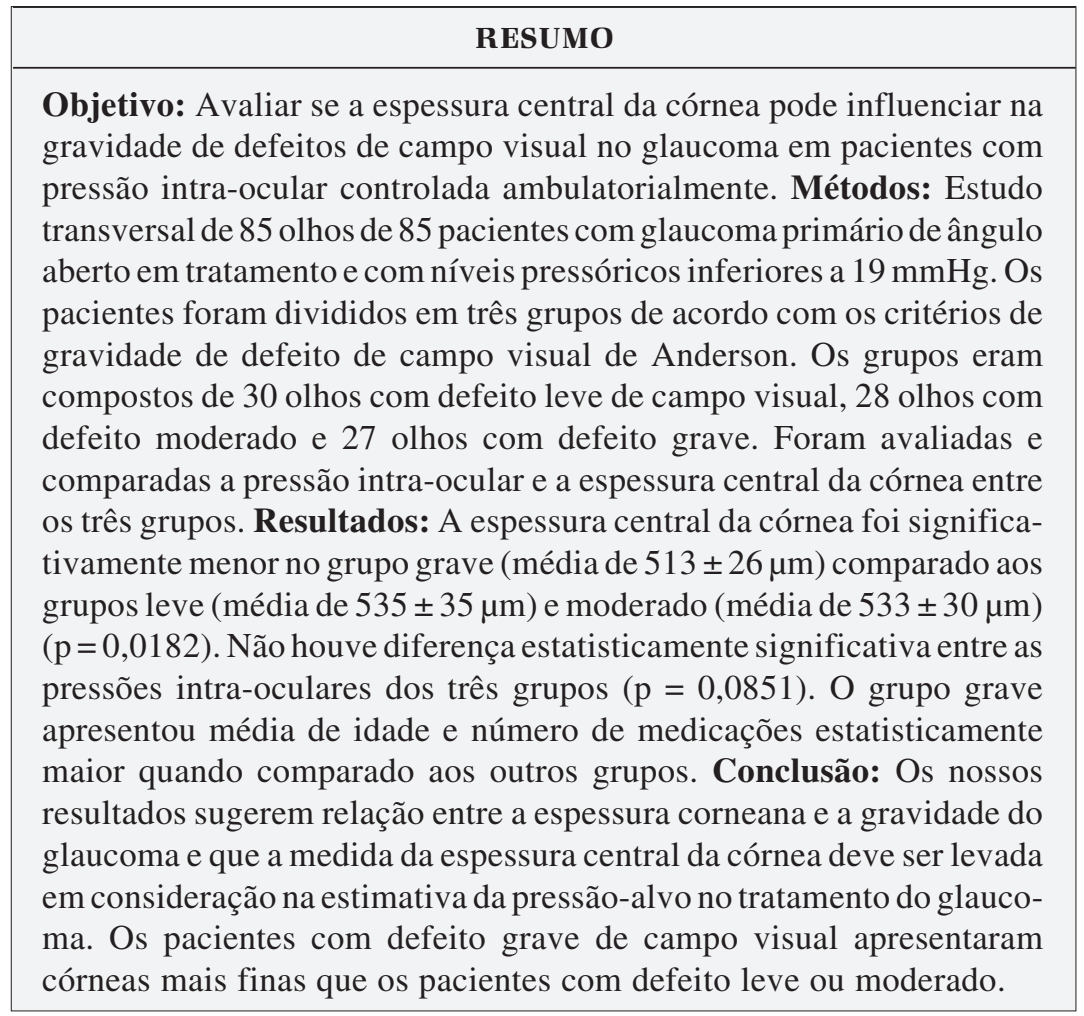

Descritores: Glaucoma de ângulo aberto; Pressão intra-ocular; Córnea; Técnicas de diagnóstico oftalmológico; Campos visuais; Tonometria ocular

\section{INTRODUÇÃO}

A tonometria de aplanação de Goldmann é influenciada pela espessura corneana. Goldmann ${ }^{(1)}$, ao desenvolver o seu tonômetro de aplanação, considerou uma espessura central da córnea média de $500 \mu \mathrm{m}$ para obtenção das medidas da pressão intra-ocular, e que apenas grandes variações da espessura corneana poderiam influenciar no resultado obtido. No entanto, tem sido observado que a medida da pressão intra-ocular obtida pelo tonômetro de aplanação pode ser subestimada em córneas finas, e superestimadas em córneas espessas ${ }^{(2)}$.

Após o advento da cirurgia a laser para miopia, foi observado que as medidas obtidas pelo tonômetro de aplanação após a cirurgia em um deter- 
minado paciente eram menores que as obtidas antes da cirurgia $^{(3)}$. Tem sido relatado também que os pacientes com glaucoma de pressão normal possuem córneas mais finas que indivíduos normais ${ }^{(4)}$ ou portadores de glaucoma primário de ângulo aberto, e que hipertensos oculares possuem córneas mais espessas $^{(5)}$. Alguns autores no entanto, apesar de encontrarem córneas mais finas em pacientes com glaucoma de pressão normal, quando comparados com pacientes portadores de glaucoma primário de ângulo aberto e indivíduos normais, não encontraram diferença estatisticamente significativa entre os três grupos $^{(6)}$. O "Ocular Hypertension Treatment Study" (OHTS) mostrou que a espessura corneana seria um fator preditivo para a conversão da hipertensão ocular em glaucoma. Pacientes com córneas mais finas apresentaram maior possibilidade de progressão para glaucoma ${ }^{(7)}$.

Alguns estudos mostraram que as medidas da pressão intra-ocular pelo tonômetro de aplanação deveriam ser ajustadas de acordo com a espessura da córnea ${ }^{(8)}$. No entanto, não existe nenhuma correlação linear que possa fazer uma correção linear da tonometria de acordo com a espessura corneana ${ }^{(9)}$.

O objetivo deste estudo é avaliar se a espessura corneana pode influenciar na gravidade do glaucoma em pacientes que apresentam pressão intra-ocular controlada ambulatorialmente.

\section{MÉTODOS}

Foram avaliados 85 pacientes ( 85 olhos) com glaucoma primário de ângulo aberto que estavam em tratamento no setor de glaucoma do Hospital Municipal da Piedade, no período de fevereiro a outubro de 2004. Os pacientes foram submetidos a exame de acuidade visual, refração, tonometria de aplanação, gonioscopia, biomicroscopia do nervo óptico, perimetria computadorizada e paquimetria ultra-sônica.

Os pacientes incluídos no estudo tinham idade maior que 35 anos, eram portadores de glaucoma primário de ângulo aberto e apresentavam pressão intra-ocular menor que $19 \mathrm{mmHg}$ nas últimas três medidas realizadas no ambulatório, durante o intervalo de 18 meses com medições da pressão intra-ocular a cada 6 meses. Todos apresentavam alterações características de glaucoma ao exame do nervo óptico e do campo visual $^{(10-11)}$. Nenhum dos pacientes poderia ter história prévia de cirurgia ocular ou procedimentos a laser.

Foram excluídos os pacientes que apresentavam outros tipos de glaucoma ou que tivessem qualquer doença intraocular que pudesse influenciar na avaliação do campo visual. Os pacientes foram divididos em três grupos de acordo com a classificação da gravidade de defeito de campo visual pelos critérios de Anderson e Pattela, em leve, moderado e grave ${ }^{(12)}$. Foram realizados pelo menos dois exames confiáveis e reprodutíveis de perimetria computadorizada no aparelho Oculus e compatíveis com a biomicroscopia do disco óptico. A estratégia usada foi Fast-Threshold no programa 24-2. Foi considerado para o estudo o olho que apresentasse glaucoma mais avançado em cada paciente. A paquimetria ultra-sônica foi realizada com aparelho DGH, sempre pelo mesmo examinador. Em cada olho foi considerada a média de cinco medidas confiáveis da espessura corneana. O trabalho foi aprovado pela Comissão de Ética do Hospital Municipal da Piedade.

Foram realizados os testes estatísticos Anova, teste F e teste de Bonferroni através do programa Bioestat. Foi considerado significativo valor de $\mathrm{p}$ menor que 0,05 .

\section{RESULTADOS}

O grupo 1 (leve) foi constituído por 30 pacientes que apresentavam defeito leve de campo visual, sendo 10 do sexo masculino e 20 do sexo feminino com média de idade de 55,8 \pm 11,6 anos. O grupo 2 (moderado) foi composto por 28 pacientes de defeito moderado de campo visual, sendo 5 de sexo masculino e 23 do sexo feminino com média de idade de 61,9 \pm 11,3 anos. O grupo 3 (grave) foi constituído por 27 pacientes com defeito grave de campo visual, sendo 14 do sexo masculino e 13 do sexo feminino com média de idade de 67,5 \pm 10,5 anos.

A tabela 1 mostra a média e o desvio padrão da idade dos três grupos havendo diferença significativa, sendo que o grupo grave apresentou idade estatisticamente maior $(\mathrm{p}=0,0012)$.

Não houve diferença significativa entre a média e o desvio padrão da pressão intra-ocular em cada grupo, sendo 14,56 \pm $2,72 \mathrm{mmHg}$ no grupo $1,15,19 \pm 2,18 \mathrm{mmHg}$ no grupo 2 e $16,08 \pm$ $2,21 \mathrm{mmHg}$ no grupo 3 , como está demonstrado na tabela 2 $(\mathrm{p}=0,0851)$.

A tabela 3 mostra a média e o desvio padrão da espessura corneana nos três grupos, sendo $535 \pm 35 \mu \mathrm{m}$ no grupo 1,533 $\pm 30 \mu \mathrm{m}$ no grupo 2 e $513 \pm 26 \mu \mathrm{m}$ no grupo 3 , mostrando que houve diferença significativa $(\mathrm{p}=0,0182)$ através do teste de análise de variância-fator único com teste de Bonferroni.

A análise comparativa entre os grupos (Tabela 4) através

\begin{tabular}{|lcc|}
\hline \multicolumn{2}{|c|}{ Tabela 1. Média e desvio padrão da idade dos três grupos } \\
Grupo & N & Idade \\
Leve & 30 & $55,8 \pm 11,6$ anos \\
Moderado & 28 & $61,9 \pm 11,3$ anos \\
Grave & 27 & $67,5 \pm 10,5$ anos \\
Valor de $p$ & $p=0,0012$ \\
Teste estatístico: Teste de análise de variância-fator único com teste de \\
Bonferroni. $p=0,05$
\end{tabular}

\begin{tabular}{|c|c|c|}
\hline Grupo & $\mathbf{N}$ & Pressão intra-ocular \\
\hline Leve & 30 & $14,56 \pm 2,72 \mathrm{mmHg}$ \\
\hline Moderado & 28 & $15,19 \pm 2,18 \mathrm{mmHg}$ \\
\hline Grave & 27 & $16,08 \pm 2,21 \mathrm{mmHg}$ \\
\hline \multicolumn{2}{|l|}{ Valor de $p$} & $p=0,0851$ \\
\hline \multicolumn{3}{|c|}{$\begin{array}{l}\text { Teste estatístico: Teste de análise de variância-fator único com teste de } \\
\text { Bonferroni. } p=0,05\end{array}$} \\
\hline
\end{tabular}


do teste de Bonferroni, mostrou que a espessura corneana dos olhos com defeito grave de campo visual, foi estatisticamente menor que a espessura corneana dos pacientes com defeito leve $(\mathrm{p}<0,005)$ e dos pacientes com defeito moderado $(\mathrm{p}<0,025)$. Não houve diferença estatisticamente significativa entre as espessuras corneanas dos grupos leve e moderado $(\mathrm{p}>0,05)$.

A tabela 5 mostra a média e o desvio padrão do número de medicações nos três grupos sendo $1,48 \pm 0,57$ medicações no grupo leve, 1,21 \pm 0,41 medicações no grupo moderado e 1,92 \pm 0,80 medicações no grupo grave, mostrando uma diferença significativa entre eles $(\mathrm{p}=0,0007)$ através do teste de análise de variância-fator único com teste de Bonferroni. $\mathrm{O}$ grupo grave apresentou maior número de medicações que os outros grupos.

\section{DISCUSS $\tilde{A} O$}

Nossos resultados mostraram relação entre a espessura corneana e a gravidade do glaucoma. Segundo o OHTS, pacientes com córneas mais finas têm maior probabilidade de desenvolverem alterações glaucomatosas ${ }^{(7)}$. O risco de conversão para glaucoma neste estudo, foi maior nos pacientes hipertensos oculares que apresentavam espessura corneana menor ou igual a $555 \mu \mathrm{m}$ comparados àqueles que apresenta-

\begin{tabular}{|c|c|c|}
\hline Grupo & $\mathbf{N}$ & Espessura corneana \\
\hline Leve & 30 & $535 \pm 35 \mu \mathrm{m}$ \\
\hline Moderado & 28 & $533 \pm 30 \mu \mathrm{m}$ \\
\hline Grave & 27 & $513 \pm 26 \mu \mathrm{m}$ \\
\hline Valor de $p$ & & $p=0,0182$ \\
\hline
\end{tabular}

\begin{tabular}{|lcc|}
\hline \multicolumn{3}{|l|}{ Tabela 4. Análise comparativa da espessura corneana por grupos } \\
Grupos & Espessura corneana & Valor de $\mathbf{p}$ \\
Leve $\times$ Moderado & $535 \mu \mathrm{m} \times 533 \mu \mathrm{m}$ & $\mathrm{p}>0,05$ \\
Leve $\times$ Grave & $535 \mu \mathrm{m} \times 513 \mu \mathrm{m}$ & $\mathrm{p}<0,005$ \\
Moderado $\times$ Grave & $533 \mu \mathrm{m} \times 513 \mu \mathrm{m}$ & $\mathrm{p}<0,025$ \\
\hline
\end{tabular}

\begin{tabular}{|lcc|}
\hline \multicolumn{2}{|l|}{ Tabela 5. Média e desvio padrão do número de medicações nos três } \\
grupos \\
Grupo & N & Número de medicações \\
Leve & 30 & $1,48 \pm 0,57$ \\
Moderado & 28 & $1,21 \pm 0,41$ \\
Grave & 27 & $1,92 \pm 0,80$ \\
Valor de $p$ & $p=0,0007$ \\
$\begin{array}{l}\text { Teste estatístico: Teste de análise de variância-fator único com teste de } \\
\text { Bonferroni. } p=0,05\end{array}$ \\
\hline
\end{tabular}

vam córneas com espessura maior que $588 \mu \mathrm{m}$. Em nosso estudo encontramos uma espessura central da córnea significativamente menor em pacientes que apresentavam defeitos graves de campo visual. No entanto, a idade do grupo de pacientes com defeitos graves de campo visual foi significativamente maior, o que também poderia influenciar na gravidade destes defeitos.

Tem sido descrita a relação entre a espessura corneana e a medida da pressão intra-ocular obtida pelo tonômetro de aplanação de Goldmann. Estudo que comparou a pressão intraocular, medida com o tonômetro de aplanação, simultaneamente com a pressão hidrostática em pacientes que seriam submetidos a cirurgia de catarata, encontrou variações na tonometria de aplanação que chegaram até mais $5 \mathrm{mmHg}$ em córneas mais espessas, e menos $5 \mathrm{mmHg}$ em córneas mais finas $^{(2)}$. Em outra pesquisa, foi descrito resultado semelhante ao ser realizada a medição da pressão intra-ocular, através da manometria e da tonometria de aplanação, com tonômetro de Perkins, e relacionadas estas medidas com a espessura corneana em pacientes que seriam submetidos à cirurgia intraocular $^{(13)}$. As córneas mais finas produziram uma subestimação da pressão intra-ocular de até $4,9 \mathrm{mmHg}$ e as córneas mais espessas, uma superestimação de até $6,8 \mathrm{mmHg}$. Outro estudo que procurou correlacionar a espessura corneana com a pressão intra-ocular de 579 pacientes, evidenciou que a pressão intra-ocular deveria ser corrigida em $\pm 2 \mathrm{mmHg}$, em $50 \%$ dos pacientes, e $\pm 3 \mathrm{mmHg}$, em $25 \%$ dos pacientes ${ }^{(8)}$. Não existe, porém, até o momento, nenhuma fórmula de regressão para corrigir a pressão intra-ocular medida com o tonômetro de aplanação de acordo com a espessura corneana ${ }^{(9)}$. Pacientes portadores de glaucoma com pressão intra-ocular aparentemente controlada e córneas mais finas, poderiam ter a sua terapêutica revista de acordo com a espessura corneana.

Estudo retrospectivo em 350 olhos de 190 pacientes, encontrou uma associação significante entre uma espessura corneana fina e dano glaucomatoso mais avançado ${ }^{(14)}$. Os autores incluíram pacientes que na consulta inicial com um especialista em glaucoma em sua instituição, apresentavam pressão intra-ocular maior que $22 \mathrm{mmHg}$ ou alterações glaucomatosas do nervo óptico com defeito correspondente de campo visual. Em nosso estudo consideramos apenas pacientes que mantinham pressão intra-ocular menor que $19 \mathrm{mmHg}$ com tratamento clínico. Utilizamos este critério para avaliar melhor a relação entre a espessura central da córnea e a gravidade do defeito de campo visual. Não encontramos diferença significativa da pressão intra-ocular nos três grupos, sugerindo uma maior influência da espessura corneana nos valores pressóricos aferidos com o tonômetro de aplanação de Goldmann.

Outros trabalhos recentes mostraram a relação entre defeitos de campo visual e a espessura corneana ${ }^{(15-16)}$. Estudo em 68 olhos de pacientes hipertensos oculares, constatou que os hipertensos oculares com córneas mais finas apresentavam defeitos de campo visual na perimetria azul-amarelo, enquanto que aqueles sem defeitos de campo visual possuíam córneas mais espessas $^{(15)}$. A avaliação de 98 olhos de 98 pacientes com 
glaucoma pré-perimétrico demonstrou maior risco de desenvolvimento de defeitos de campo visual naqueles pacientes que possuíam córneas mais finas ${ }^{(16)}$. Em outro estudo, com 65 pacientes com hipertensão ocular e 52 indivíduos com olhos normais, foi constatado que hipertensos oculares que apresentavam defeitos de campo visual através da perimetria de freqüência dupla possuíam córneas mais finas comparados aos hipertensos oculares que apresentavam resultados normais neste exame ${ }^{(17)}$.

Os pacientes do grupo grave, em nosso estudo, possuíam idade significativamente mais elevada e faziam uso de maior número de medicações para controle do glaucoma em relação aos outros dois grupos. O OHTS constatou que pacientes mais idosos apresentavam córneas mais finas ${ }^{(7)}$. O maior número de medicações no grupo grave pode ser explicado pela necessidade de uma terapêutica mais agressiva nesses pacientes para controle do glaucoma.

Não houve diferença estatisticamente significativa nos valores da espessura corneana entre os grupos leve e moderado. Este dado sugere que apenas a partir de um determinado grau de gravidade a espessura corneana seria um fator de risco para o desenvolvimento dos defeitos de campo visual. Apesar de que a variação da espessura corneana em uma curva diária de 24 horas não parecer influenciar na tonometria, realizamos todas as medições da espessura corneana no mesmo horário, entre 14:30 e 15:30 horas $^{(18-19)}$.

$\mathrm{O}$ achado de que pacientes com glaucoma mais avançado possuem córneas mais finas sugere que a pressão intra-ocular alvo deve ser revista neste grupo com espessura corneana menor. O "Advanced Glaucoma Intervention Study" (AGIS) constatou que pacientes com glaucoma avançado têm menor possibilidade de progressão dos defeitos de campo visual com pressões abaixo de $12 \mathrm{mmHg}^{(20)}$. No entanto, a medida da espessura corneana não foi levada em conta na estimativa da pressão intra-ocular neste trabalho. Um estudo com 188 pacientes com glaucoma ou suspeita de glaucoma, constatou que $55,9 \%$ destes pacientes deveriam ter a sua conduta modificada de acordo com a espessura corneana ${ }^{(21)}$.

\section{CONCLUSÕES}

Os nossos resultados sugerem uma relação entre a espessura corneana e a gravidade do glaucoma e que a medida da espessura central da córnea deve ser levada em consideração na estimativa da pressão alvo no tratamento do glaucoma. Os pacientes com glaucoma grave apresentaram córneas mais finas que os pacientes com glaucoma leve ou moderado.

\section{ABSTRACT}

Purpose: To assess whether central corneal thickness is related to glaucomatous visual field loss severity among patients with office-controlled intraocular pressure in the normal ran- ge. Methods: This transversal study included 85 eyes of 85 patients with treated primary open-angle glaucoma and documented intraocular pressure below $19 \mathrm{mmHg}$. The patients were divided into three groups according to Anderson criteria of field abnormality. The groups were composed of 30 eyes with mild defect, 28 eyes with moderate defect and 27 eyes with severe defect. Intraocular pressure and central cornea thickness were compared and evaluated between the three groups. Results: Central corneal thickness was statistically thinner in the severe group (mean $=513 \pm 26 \mu \mathrm{m}$ ) compared with the mild defect group (mean $=535 \pm 35 \mu \mathrm{m})$ and the moderate group $($ mean $=533 \pm 30 \mu \mathrm{m})(\mathrm{p}=0.0182)$. There was no statistically significant difference between intraocular pressures in the three groups $(\mathrm{p}=0.0851)$. The severe group showed a statistically higher age and number of medications than the other groups. Conclusions: The central corneal thickness measurement is desirable when establishing target intraocular pressure of patients with open-angle glaucoma. Central corneal thickness is a clinical factor in determining glaucoma severity. Patients with severe field loss showed lower corneal thickness compared with patients with mild and moderate visual field loss.

Keywords: Glaucoma, open angle; Intraocular pressure; Cornea; Diagnostic techniques, ophthalmological; Visual fields; Tonometry, ocular

\section{REFERÊNCIAS}

1. Goldmann H, Schmidt TH. Uber applanations tonometrie. Ophthalmologic. 1957;134(4):221.

2. Ehlers N, Bramsen T, Sperling S. Applanation tonometry and central corneal thickness. Acta Ophthalmol (Copenh). 1975;53(1):34-43.

3. Fontes BM, Navajas EV, Melo LAS Jr, Fontes MLM, Fontes PC. Alteração da pressão intra-ocular aferida pela tonometria de aplanação após laser in situ keratomileusis. Rev Bras Oftalmol. 2004;63(5-6):315-20.

4. Morad Y, Sharon E, Hefetz L, Nemet P. Corneal thickness and curvature in normal-tension glaucoma. Am J Ophthalmol. 1998;125(2):164-8. Comment in Am J Ophthalmol. 1998;126(2):326-8.

5. Hansen FK, Ehlers N. Elevated tonometer readings caused by a thick cornea Acta Ophthalmol (Copenh). 1971;49(5):775-8

6. Meirelles SHS, Stohler NSF, Mariano MP, Yamane R, Yamane Y. Espessura corneana no glaucoma de pressão normal. Rev Bras Oftalmol. 2001;60(1):56-64.

7. Kass MA, Heuer DK, Higginbotham EJ, Johnson CA, Keltner JL, Miller JP, et al. The Ocular Hypertension Treatment Study: a randomized trial determines that topical ocular hypotensive medication delays or prevents the onset of primary open-angle glaucoma. Arch Ophthalmol. 2002;120(6):701-13; discussion 829-30. Comment in: Arch Ophthalmol. 2003;121(7):1070; author reply 1070. Arch Ophthalmol. 20041;122(7):1088-9; author reply 1089.

8. Stodmeister R. Applanation tonometry and correction according to corneal thickness. Acta Ophthalmol Scand. 1998;76(3):319-24.

9. Feltgen N, Leifert D, Funk J. Correlation between central corneal thickness, applanation tonometry, and direct intracameral IOP readings. Br J Ophthalmol 2001;85(1):85-7. Comment in: Br J Ophthalmol. 2002;86(1):120-1.

10. Jonas BJ, Budde WM, Panda-Jonas S. Ophthalmoscopic evaluation of the optic nerve head. Surv Ophthalmol. 1999;43(4):293-320.

11. Comparison of glaucomatous progression between untreated patients with normal-tension glaucoma and patients with therapeutically reduced intraocular pressures. Collaborative Normal-Tension Glaucoma Study Group. Am J Ophthalmol. 1998;126(4):487-97. Erratum in: Am J Ophthalmol 1999;127(1):120. Erratum in: Am J Ophthalmol 1999;127(1):120.

12. Anderson D, Pattela VM. Automated static perimetry. St. Louis: Mosby; 1999 
13. Whitacre MM, Stein RA, Hassanein K. The effect of corneal thickness on applanation tonometry. Am J Ophthalmol, 1993;115(5):592-6.

14. Herndon LW, Weizer JS, Stinnett SS. Central corneal thickness as a risk factor for advanced glaucoma damage. Arch Ophthalmol. 2004;122(1):17-21.

15. Medeiros FA, Sample PA, Weinreb RN. Corneal thickness measurements and visual function abnormalities in ocular hypertensive patients. Am J Ophthalmol. 2003;135(2):131-7.

16. Medeiros FA, Sample PA, Zangwill LM, Bowd C, Aihara M, Weinreb RN. Corneal thickness as a risk factor for visual field loss in patients with preperimetric glaucomatous optic neuropathy. Am J Ophthalmol. 2003;136(5):805-13.

17. Medeiros FA, Sample PA, Weinreb RN. Corneal thickness measurements and frequency doubling technology perimetry abnormalities in ocular hypertensive eyes. Ophthalmology 2003;110(10):1903-8.
18. Shah S, Spedding C, Bhojwani R, Kwartz J, Henson D, Mc Leod D. Assessment of the diurnal variation in central corneal thickness and intraocular pressure of patients with suspected glaucoma. Ophthalmology 2000;107(6):1191-3.

19. Cronemberger S, Calixto N, Costa LT, Soares FM. Corneal thickness and daily curve of intraocular pressure in suspected and glaucomatous patients. Arq Bras Oftalmol. 2005;68(2):185-8.

20. The Advanced Glaucoma Intervention Study (AGIS): 7. The relationship between control of intraocular pressure and visual field deterioration. The AGIS Investigators. Am J Ophthalmol. 2000;130(4):429-40. Comment in: Am J Ophthalmol. 2000;130(4):490-1.

21. Shih CY, Zizin JSG, Trokel SL, Tsai JC. Clinical significance of central corneal thickness in the management of glaucoma. Arch Ophthalmol. 2004;122(9):1270-5.

\title{
Bula Resumida do Produto
}

\author{
Xalatan $\circledast$ \\ (latanoprosta)
}

Xalatan ${ }^{\circledR}$ (latanoprosta) é um análogo da prostaglandina $F 2 \alpha$ que reduz a pressão intra-ocular (PIO)aumentando a drenagem do humor aquoso. Indicações: redução da pressão intra-ocular (PIO) elevada em pacientes com glaucoma de ângulo aberto e hipertensão ocular. Contra-indicações: hipersensibilidade a latanoprosta ou a qualquer componente da fórmula. Advertências e precauções: pode ocorrer alteração gradual da coloração do olho pelo aumento da pigmentação da íris. As lentes de contato devem ser removidas antes da instilação do colírio e podem ser recolocadas após 15 minutos (vide bula completa do produto). Uso durante a gravidez e lactação: Xalatan ${ }^{\circledR}$ deve ser usado durante a gravidez apenas se o benefício previsto justificar o risco potencial para o feto e deve ser utilizado com cautela em mulheres lactantes. Interações medicamentosas: estudos in vitro mostraram que ocorre precipitação quando colírios contendo timerosal são misturados com Xalatan®. Também se observou em estudos que o efeito redutor da pressão intra-ocular da latanoprosta é aditivo ao do timolol, dipivalilepinefrina, acetazolamida e pilocarpina. Se tais produtos forem utilizados, o colírio deve ser administrado com um intervalo de, no mínimo, 5 minutos (vide bula completa do produto). Reações adversas: nos estudos clínicos as seguintes reações foram relatadas: irritação ocular, blefarite, hiperemia conjuntival, dor ocular, aumento da pigmentação da íris, erosões epiteliais puntiformes transitórias, edema de pálpebra, e rash cutâneo. Após comercialização foram relatadas as seguintes reações: tontura e cefaléia, edema e erosões da córnea, conjuntivite, alteração nos cílios e lanugem da pálpebra, irite/uveíte, ceratite, edema macular incluindo edema macular cistóide, cílios irregulares que podem causar irritação ocular, visão embaçada, asma, agravamento da asma, crises agudas de asma, dispnéia, escurecimento da pele da pálpebra, reação cutânea local na pálpebra, dor muscular/articulação (vide bula completa do produto). Posologia: 1 gota no(s) olho(s) afetado(s), uma vez ao dia. Se o paciente estiver usando outro colírio, este deve ser utilizado pelo menos 5 minutos após o uso de Xalatan®. Superdosagem: se ocorrer superdosagem, deve-se instituir tratamento sintomático. Apresentação: solução oftálmica estéril $50 \mathrm{mcg} / \mathrm{mL}$ em embalagem com frasco gotejador de 2,5 mL. USO ADULTO.USO OFTÁLMICO. VENDA SOB PRESCRIÇÃO MÉDICA. Para maiores informações, consulte a bula completa do produto (xat02). Documentação científica e informações adicionais estão à disposição da classe médica mediante solicitação. Laboratórios Pfizer Ltda., Rua Alexandre Dumas, 1860 - Chácara Santo Antônio, São Paulo, SP - CEP 04717-904. Tel.: 0800-16-7575. Xalatan®. MS - 1.0216.0129. 